\title{
Mission et civilisation dans l'Ouest canadien : Vital Grandin, 1829-1902
}

\section{Claude Champagne}

Volume 50, numéro 1, 1983

Bilan de l'histoire religieuse au Canada

Canadian Catholic History: A survey

URI : https://id.erudit.org/iderudit/1007050ar

DOI : https://doi.org/10.7202/1007050ar

Aller au sommaire du numéro

Éditeur(s)

Les Éditions Historia Ecclesiæ Catholicæ Canadensis Inc.

ISSN

0318-6172 (imprimé)

1927-7067 (numérique)

Découvrir la revue

Citer cet article

Champagne, C. (1983). Mission et civilisation dans l'Ouest canadien : Vital

Grandin, 1829-1902. Sessions d'étude - Société canadienne d'histoire de l'Église catholique, 50(1), 341-359. https://doi.org/10.7202/1007050ar

Tous droits réservés @ Les Éditions Historia Ecclesiæ Catholicæ Canadensis Inc., 1983
Ce document est protégé par la loi sur le droit d'auteur. L'utilisation des services d'Érudit (y compris la reproduction) est assujettie à sa politique d'utilisation que vous pouvez consulter en ligne.

https://apropos.erudit.org/fr/usagers/politique-dutilisation/ 


\section{Mission et civilisation dans l'Ouest canadien: Vital Grandin, 1829-1902}

Les missionnaires catholiques auprès des Métis et des Indiens de l'Ouest canadien, au siècle dernier, ont compris leur travail d'abord comme évangélisation et christianisation mais aussi comme oeuvre de civilisation. C'est cette notion que nous chercherons à éclaircir ici à travers un exemple-type, $\mathbf{M}^{\mathrm{gr}}$ Vital Grandin, o.m.i., missionnaire dans l'Ouest à partir de 1854 , évêque coadjuteur de $\mathbf{M}^{\mathrm{gr}}$ Alexandre Taché en 1859 et évêque titulaire du diocèse de Saint-Albert de 1871 à sa mort, en 1902 . $\mathrm{M}^{\mathrm{gr}}$ Grandin fut un témoin privilégié et un des principaux acteurs de l'activité missionnaire dans le Nord-Ouest canadien au XIX ${ }^{\mathrm{e}}$ siècle. Après avoir mis en lumière la notion de civilisation de Grandin et de ses collaborateurs, nous examinerons l'effort de civilisation des adultes indiens et métis, les difficultés rencontrées, leur réaction au choc de la culture occidentale. Nous étudierons enfin le travail de civilisation des Indiens par leurs enfants.

Pour Grandin, «civilisation» signifiait faire passer les Indiens et les Métis de leur état originel à un état considéré comme plus évolué; les former au travail, principalement agricole, à la vie de famille et à la vie "civilisée», c'est-à-dire celle vécue en Europe ${ }^{1}$. Mais l'évêque de SaintAlbert distingue entre la vraie et la fausse civilisation qui progressait de son temps en Europe, celle qui rejetait à la fois l'autorité de Dieu et l'autorité civile ${ }^{2}$. La civilisation ne pouvait être authentique sans la religion catholique qui a contribué à son apparition en Europe.

\footnotetext{
${ }^{1}$ Rapport de Vital Grandin au cardinal Jean Simeoni, secrétaire de la Propagande de 1868 à 1875 et préfet de 1878 à 1892, 20 octobre 1880, Écrits de Grandin [désormais EG], vol. 7, P. 415. Les Écrits de $\mathrm{M}^{\mathrm{gr}}$ Grandin n'ont pas été publiés. Ils sont conservés à la Postulation générale des Oblats, à Rome, aux Archives diocésaines d'Edmonton, de Saint-Boniface et du Keewatin de même qu'aux Archives Deschâtelets d'Ottawa. Une copie dactylographiée en 26 volumes existe, cependant, depuis 1966. On en trouve une, notamment, au Centre de Recherche de l'Université Saint-Paul.

${ }^{2}$ Adresse de Grandin à Sir John Campbell, gouverneur-général du Canada de 1878 à 1883 , s.d. (EG 21, 520).
} 
Les missionnaires de Grandin étaient très conscients de l'importance du travail de civilisation auprès de leurs fidèles. Déjà leur fondateur, $\mathbf{M}^{\mathrm{gr}}$ Eugène de Mazenod, dans son Instruction relative aux missions étrangères, les invitait à travailler en ce sens:

Loin de considérer le travail de formation des sauvages aux nécessités de la vie sociale comme étranger à leur programme, les membres de la Société y verront, au contraire, un excellent moyen de contribuer au bien de la Mission et de rendre plus fructueux leur apostolat. C'est pourquoi ils ne négligeront rien pour amener les tribus nomades à renoncer à leurs habitudes de vie errante et à se choisir des emplacements où ils apprendront à bâtir des maisons, à cultiver la terre et à se familiariser avec les premiers arts de la civilisation. ${ }^{3}$

Pour de Mazenod, il s'agissait de former les Indiens aux nécessités de la vie sociale. Pour ce faire, il fallait amener les tribus nomades à renoncer à leur vie errante, à se choisir un emplacement où ils apprendraient à bâtir des maisons, à cultiver la terre et à se familiariser avec les premiers arts de la civilisation, c'est-à-dire les différents métiers. Or, deux moyens s'offraient aux missionnaires pour cette oeuvre de civilisation: "la religion comme le meilleur moyen de civiliser et l'école comme moyen secondaire" ${ }^{4}$. La religion était l'élément civilisateur essentiel mais ce travail s'appuyait sur l'éducation et les écoles.

Avec l'annexion des Territoires du Nord-Ouest au Canada, en 1870, le dilemne devint crucial pour les missionnaires. Face au choc de l'arrivée des Blancs, ils pouvaient chercher à isoler Indiens et Métis pour les préserver, mais alors ces derniers ne seraient pas qualifiés pour la société technologique de l'avenir, ou ils pouvaient contribuer à l'assmilation et à l'intégration des Indiens et des Métis à la société blanche, détruisant ainsi leur culture traditionnelle.

Dès 1870 , Grandin pressentait les conséquences désastreuses de l'arrivée des immigrants pour les Métis et les Indiens.

Des émigrants vont nous venir de toute part et les villes vont s'élever comme par enchantement au milieu de nos plaines et de

\footnotetext{
${ }^{3}$ Claude Champagne, "Instruction de $\mathrm{M}^{\mathrm{gr}}$ de Mazenod relative aux missions étrangères", Kerygma 25 (1975) p. 175.

4 Notes sur les Missions: Etablissements, difficultés jusqu'en septembre 1886 (EG 6, 359).
} 
nos forêts et d'après toute probabilité nos pauvres sauvages peutêtre même nos Métis devront se retirer dans l'extrême nord si même la civilisation ne les tue pas tout à fait. ${ }^{5}$

\section{LA CIVILISATION DES ADULTES MÉTIS ET INDIENS}

La décennie 1870-1880 allait voir les missionnaires travailler à préparer leurs chrétiens à affronter le choc de l'arrivée des colons, choc qui se fit sentir dans le diocèse de Saint-Albert plus particulièrement à partir de 1880. On optait pour l'intégration des Indiens et des Métis au monde des Blancs, malgré le risque de détruire leur culture. Dès cette époque, les Oblats préparaient le passage des Métis d'origine canadiennefrançaise d'une vie nomade peu favorable tant à la christianisation qu'à la civilisation ${ }^{6}$ à une vie d'agriculteur en mettant sur pied une ferme à saint-Albert.

Mais nous ne nous occupons pas seulement de faire de nos gens des chrétiens et des savants, nous voulons aussi leur apprendre à cultiver et pour cela nous prêchons surtout d'exemple. Nous avons ici une ferme considérable, nous ensemençons chaque année de trente à quarante arpents de terre. ${ }^{7}$

$\mathrm{Au}$ Chapitre Général de 1873, Grandin revint sur la question. Le passage du nomadisme et de la chasse à une vie sédentaire devenait urgent.

Dans notre mission de Saint-Albert, nos Pères ne s'occupent pas seulement de former des chrétiens, ils doivent aussi y former des agriculteurs; les métis jusqu'à présent ne vivaient guère que du produit de la chasse; mais, cette ressource ayant considérablement diminué et pouvant d'un jour à l'autre leur faire complètement défaut, il a fallu leur en procurer une bien plus abondante et surtout plus certaine, c'est celle qu'un sol riche et vaste peut leur fournir; mais, pour amener les métis à cultiver la terre, il a fallu leur en donner l'exemple. ${ }^{8}$

\footnotetext{
${ }^{5}$ Grandin à M. Latouche, 23 décembre 1870 (EG 25, 370).

${ }^{6}$ Grandin à M. et Mme Latouche, 8 juillet 1855 (EG 25, 251-52).

${ }^{7}$ Grandin au P. Pierre Aubert, assistant-général de 1867 à 1887, 7 décembre 1872 (EG 14, 539).

${ }^{8}$ Rapport de Grandin sur le Vicariat du diocèse de Saint-Albert (EG 13, 345).
} 
Grandin ne pensait pas qu'aux Métis, considérés comme demicivilisés ${ }^{9}$, mais aussi aux Indiens, et pendant son séjour en Europe pour participer au Chapitre de 1873, il prévoyait établir au lac d'Orignal une ferme pour eux.

Je me propose, à mon retour d'Europe, essayer d'établir là une espèce de ferme modèle où des hommes dévoués que la congrégation des Oblats me prépare ad hoc essayeront d'apprendre la culture aux sauvages les mieux disposés, ensemenceront leurs terres, les aideront à bâtir des maisons et peut-être à force de dévouement et de sacrifices finirons-nous par les civiliser un peu. ${ }^{10}$

Civiliser signifiant donc initier à la vie agricole et à la connaissance des métiers nécessaires à la construction des maisons. À la même époque, le Gouvernement canadien signa des traités avec les différentes tribus indiennes. Or, Grandin s'est montré très sympathique aux traités qui fixaient les Indiens sur les réserves, faisant ainsi obstacle au nomadisme.

Il [le traité] leur [les Indiens] est certainement bien favorable et il ne met aucun obstacle à la religion, j'espère même qu'avec ce traité il nous sera beaucoup plus facile de civiliser et de christianiser les sauvages. ${ }^{11}$

Les missionnaires catholiques ont rendu le traité possible et y voyaient des avantages tant pour les Indiens que pour leur activité missionnaire, ce que les Indiens leur reprochèrent plus tard.

Les reproches que nous font fréquemment les vieux sauvages et que pour ma part j'ai eu souvent à supporter sont là pour prouver ce que j'affirme (les missionnaires catholiques ont rendu le traité possible). C'est vous, nous disent-ils, qui êtes cause que les blancs se sont emparés de nos terres, que nous nous sommes liés par un traité avec eux, ce à quoi nous n'aurions jamais consenti sans vous. ${ }^{12}$

\footnotetext{
${ }^{9}$ Grandin à $\mathrm{M}^{\mathrm{gr}}$ Ignace Bourget, évêque de Montréal, 25 avril 1876 (EG 8, 183). Giraud souligne qu'en dépit de l'exemple des missionnaires, la vie agricole ne dépassa jamais le stade de quelques défrichements élémentaires autour des demeures métisses. Cf. Marcel Giraud, Le Métis Canadien. Son rôle dans l'histoire des provinces de l'Ouest, $\mathrm{Pa}$ ris, 1945, pp. 1075-6. (Travaux et mémoires de l'Institut d'Ethnologie 44).

${ }^{10}$ Diocèse de Saint-Albert: Missions sauvages avant le traité (désormais DSA) (EG 6, 210-11).

${ }^{11}$ Grandin à M. Latouche, 16 décembre 1876 (EG 25, 456).

12 DSA (EG 6, 211-2).
} 
Assez rapidement pourtant, une conclusion s'imposa: il était difficile, sinon impossible de "civiliser" les adultes. Parlant du travail du Gouvernement canadien, Grandin fit part de ses conclusions. Il émettait des réserves sur la possibilité de faire des Indiens des agriculteurs.

Il [le Gouvernement] voudrait aussi les [Indiens] empêcher de mourir de faim. Pour cela, il leur facilite la culture, leur fournit des charrues et des boeufs, et fait d'énormes dépenses, qui resteront, hélas! sans résultat. Les sauvages adultes ne pourront jamais s'astreindre à la culture; c'est un état trop contraire à leur manière de vivre. ${ }^{13}$

Il exprimait cette conviction en 1879 , c'est-à-dire quelques années après son propre projet de ferme modèle au moment où le Gouvernement établissait les réserves et donnait aux Indiens des fermiers-instructeurs ${ }^{14}$. Il revenait sur la même constatation en 1883 .

Des sauvages qui jusqu'à présent n'ont eu entre les mains que le fusil et la flèche, les obliger pour vivre à se servir de la pioche et de la charrue, c'est ni plus ni moins les condamner à mort. ${ }^{15}$

Grandin constatait à la fois la disparition du produit de la chasse et de la pêche et l'impossibilité pour les Indiens adultes de passer à l'agriculture. L'évêque proposait alors un autre plan au Gouvernement pour préserver certains Indiens et leur mode de vie en les isolant des étrangers.

Je propose un plan au Gouvernement qui ne sera jamais accepté. $[\ldots]$ Ce sera l'éloignement de tous ces traiteurs libres qui sont en réalité la cause de l'appauvrissement du pays et des sauvages. Si une compagnie unique, sous un sage et puissant contrôle avait le droit de commerce dans les pays du Nord qui ne sont pas susceptibles d'être colonisés, ce serait son intérêt de veiller à ce que les animaux dont les sauvages vivent ne fussent point entièrement détruits; elle pourrait, pour avoir des peaux ou des provisions plus longtemps, renoncer à en avoir pendant quelques années, tandis que les choses étant ce qu'elles sont, chacun tirera des sauvages ce qu'il pourra tirer et quand ces pauvres malheureux auront tué le

\footnotetext{
${ }^{13}$ Grandin à $\mathrm{M}^{\mathrm{gr}}$ Edouard-Charles Fabre, évêque de Montréal, ler mai 1879 (EG 8, 278).

${ }^{14}$ Dès 1877 , le Gouvernement fournissait semences et instructeurs aux Indiens. Si les rapports des officiers étaient optimistes en 1880, il fallut reconnaître l'échec et fermer les fermes avant 1885. Cf. Morris Zaslow, The Opening of the Canadian North 1870-1914, Toronto-Montréal, 1971, p. 19, (The Canadian Centenary Series 16).

${ }^{15}$ Grandin à l'Archevêque de Québec et aux Évêques de la Province de Québec, 29 janvier 1883 (EG 7, 495).
} 
dernier caribou et le dernier renard, on les laissera mourir de faim et de froid tandis qu'une compagnie jouissant chez eux du droit de commerce à l'exclusion de tout autre, pourrait bien avoir des obligations envers les vieillards, les orphelins et les pauvres sauvages auxquels la vie nomade n'est plus possible. ${ }^{16}$

Ce plan d'isoler les Indiens des Blancs, de préserver leur mode de vie et leurs ressources visait particulièrement les Montagnais du district de la rivière aux Anglais, partie non-colonisable du territoire. Nous n'avons pas d'indices que cette proposition ait trouvé écho auprès du Gouvernement du Nord-Ouest.

Pour l'évêque de Saint-Albert, les Indiens n'avaient pas grand avenir. "L'on peut dire que ce diocèse, s'il conservait les mêmes habitants, serait sans avenir, comme toutes les contrées peuplées par les sauvages de l'Amérique du Nord" ${ }^{17}$. L'impact de l'arrivée des Blancs, souvent immoraux, menaçait les Indiens de disparition, en particulier les PiedsNoirs ${ }^{18}$.

Grandin et ses Oblats furent témoins des difficultés croissantes des Métis et des Indiens face à l'arrivée de la «civilisation» des Occidentaux, ce que George Stanley appelle un "problème de frontière" ${ }^{19}$. Non seulement furent-ils témoins mais aussi acteurs puisqu'ils intervinrent fréquemment auprès du Gouvernement pour signaler les difficultés et les injustices. Déjà en 1873, l'évêque de Saint-Albert percevait les tensions existant entre les Métis, les Indiens et le Gouvernement, de même que l'exploitation de la simplicité des Métis par les nouveaux venus: vol des terres des premiers occupants, etc... Si $\mathbf{M}^{\mathrm{gr}}$ Grandin était prêt à appuyer les droits des Métis, il ne pouvait encourager la révolution contre l'autorité légitime. On ne put pourtant éviter la rébellion de $1885^{20}$.

\footnotetext{
${ }^{16}$ Grandin à $\mathrm{M}^{\mathrm{gr}}$ Alexandre Taché, archevêque de Saint-Boniface, 9 juillet 1880 (EG $10,176)$; voir aussi Grandin à l'hon. David Laird, superintendant du Nord-Ouest, "Indian Commissioner for the prairies ", lieutenant-gouverneur des Territoires de 1876 à 1881, S.D. (EG 22, 201-2).

${ }^{17}$ Rapport de Grandin sur le Vicariat (EG 13, 249-50).

${ }^{18}$ Grandin au P. Joseph Fabre, supérieur général, 6 avril 1889 (EG 13, 502-3).

${ }^{19}$ George F.G. Stanley, The Birth of Western Canada. A History of the Riel Rebellions, Toronto, 1966, p. vii.

${ }^{20}$ Claude Champagne, Mission et Église chez Mgr Vital Grandin, o.m.i. (1829-1902), Thèse doctorale à la Faculté de Missiologie de l'Université Pontificale Grégorienne, Rome, 1982, pp. 315-27.
} 
Si le Gouvernement protégeait les Indiens sur les réserves, la situation des Métis n'a fait qu'empirer après la rébellion de 1885. En juillet 1898, Grandin traçait le portrait de ceux qui s'étaient éloignés des centres et de ceux qui y étaient demeurés. Certains ont choisi de s'éloigner.

Les métis descendants des meilleures familles, sont pauvres; ils ont vendu toutes leurs terres devenues aujourd'hui la propriété des commerçants qui ne craignaient pas de leur vendre à crédit tant qu'ils pouvaient hypothéquer quelques propriétés; n'ayant plus rien, ils s'éloignent, vont se fixer là où la civilisation n'est point encore parvenue, je dis civilisation, pour être plus juste, je devrais dire civilisation moderne. Ils seront tranquilles dans ces places éloignées où ils peuvent encore chasser et pêcher et cultiver au moins des patates et de l'orge. ${ }^{21}$

Grandin précise ici que la civilisation des immigrants blancs est la civilisation moderne. Quant aux Métis demeurés près des grandes villes, leur condition était plus pénible.

Ceux qui ne s'éloignent pas végètent autour de nos nouvelles villes, tâchant de gagner leur vie comme ils peuvent en travaillant, trop s'adonnent à l'ivrognerie et à la débauche. Cette population $[\ldots]$ croit pouvoir se permettre ce que les blancs font malgré les lois, la police, les juges, la prison et la corde: les crimes se multiplient. Nous avons ici des familles où de grands garçons n'ont plus aucun respect pour leurs pères et leurs mères, surtout qui volent les biens de la famille et des voisins pour se procurer de la boisson. ${ }^{22}$

Face à cette situation, le P. Albert Lacombe, avec l'encouragement de $\mathrm{M}^{\mathrm{gr}}$ Grandin et des évêques de Saint-Boniface et de Prince-Albert, tenta de former, dans la région de la réserve indienne de Saddle Lake, une colonie destinée aux Métis, Saint-Paul-des-Métis ${ }^{23}$. L'évêque de Saint-Albert fit appel aux Métis et mit en évidence leurs talents et leurs qualités.

Ayez confiance en vous-mêmes, sans orgueil, vous valez bien les autres et dites-vous: ces Allemands, ces Polonais, ces Ménonites

\footnotetext{
${ }^{21}$ Souvenirs (EG 4, 178). Voir la situation des Métis après l'insurrection: Giraud, Le Métis, pp. 1211-30.

22. Souvenirs (EG 4, 178).

${ }^{23}$ Voir Emeric 0. Drouin, Joyau dans la plaine: Saint-Paul, Alberta, colonie métisse 1896-1909, paroisse blanche 1909-1951, Québec, 1968, et Giraud, Le Métis pp. 1225-29 et 1264 .
} 
[Mennonites], ces étrangers qui arrivent dans notre pays ne le connaissent pas, ils arrivent pauvres souvent, déguenillés, méprisés, et cependant à force d'énergie, de patience, d'économie, ils se tirent d'affaire. Ne les valez-vous pas ces gens-là? Vous avez sur eux l'avantage de connaître le pays, et bien d'autres avantages encore. Je suis convaincu que si vous êtes courageux et énergiques et surtout bons chrétiens, votre colonie deviendra vite une colonie modèle. ${ }^{24}$

Maiheureusement, à cause de leur défiance envers le clergé depuis la rébellion de 1885 condamnée par l'Église, la réponse des familles métisses fut plutôt lente. Malgré la prévention des Métis contre le clergé, Grandin reconnaissait toujours à l'Église une responsabilité face à la situation des Métis; on ne pouvait laisser végéter ceux pour qui le choc de l'arrivée des Blancs avait été si dévastateur. Si l'Église n'avait pu réussir à préparer les Indiens et les Métis à affronter la civilisation moderne, elle convenait que c'était une partie de sa mission de «sauver», de "racheter» les Métis ${ }^{25}$. Ceux-ci ne répondirent pas comme on l'avait espéré à l'initiative de Lacombe. L'incendie de l'école de Saint-Pauldes-Métis par des élèves vint mettre un terme à cette tentative au début de $1905^{26}$. Giraud, qui conclut son étude en traitant de la situation des Métis vers 1935-1936, y parle de la désagrégation qui a souvent pris l'apparence d'une agonie physique et morale ${ }^{27}$.

\section{LA CIVILISATION DES INDIENS PAR LEURS ENFANTS}

Dès le début de leur activité missionnaire, Grandin et ses confrères ont manifesté leur intérêt pour l'éducation des enfants. C'est en voulant préparer Métis et Indiens au choc de l'arrivée des Blancs, dans les années 1870, qu'ils conclurent à l'impossibilité de civiliser les adultes et décidèrent d'orienter leurs efforts civilisateurs vers les enfants. L'expérience des orphelinats et des premières écoles de mission appuyait l'opinion de Grandin: pour sauver les Indiens, il fallait les prendre très jeunes, les élever et en faire des hommes demi-civilisés - c'est-à-dire l'équivalent des Métis ou des colons ordinaires - auxquels la vie sauvage ne serait plus possible ${ }^{28}$.

\footnotetext{
${ }^{24}$ Grandin aux Métis résidant à la mission St-Paul-des-Métis, 25 juin 1897 (EG 12, 554).

${ }^{25}$ Documents collectifs des évêques de la province de Saint-Boniface, 7 mars 1899 (EG $17,477-478$ ).

${ }^{26}$ Drouin, Joyau, pp. 233-50.

${ }^{27}$ Giraud, Le Métis, pp. 1250-86.

${ }^{28}$ Grandin à $\mathrm{M}^{\mathrm{gr}}$ Bourget, 25 avril 1876 (EG 8, 182-3).
} 
Je ne puis certifier que ces enfants à quelque nation qu'ils appartiennent ne sont plus sauvages en sortant de chez nous. [...] Parmi les Métis et colons ordinaires de nos pays ils peuvent tenir leur place avec honneur. L'expérience prouve que la civilisation tue les sauvages et les fait disparaître; plus de 20 années d'expérience me prouvent à moi que notre manière de civiliser ne tue pas. [...] En sortant de nos maisons ces enfants n'ont plus rien des sauvages que le sang; ils ont même oublié leur langue naturelle [maternelle] si bien que la vie sauvage ne leur est plus possible; nous leur inspirerons pour ce genre de vie un dégoût prononcé, en sorte qu'ils sont humiliés quand on leur rappelle leur origine. ${ }^{29}$

Grandin et ses collaborateurs visaient donc à faire disparaître chez les Indiens leur aspect "sauvage" en éliminant leur langue maternelle et en rendant impossible le retour à leur genre de vie. Mais ce travail de civilisation ne se révélait possible qu'en prenant les Indiens petits enfants ${ }^{30}$.

Mais alors que penser de la contribution des Obats aux études de linguistique et d'ethnologie ${ }^{31}$ ? Les Oblats étaient convaincus de la nécessité de proclamer la Bonne Nouvelle aux populations indiennes en leur langue ${ }^{32}$. Mais Grandin ne semble pas tellement vouloir conserver la langue indienne: il y aurait tellement d'avantages à ce que les Indiens parlent français. Il faut ajouter que d'autres missionnaires, comme le P. Grollier, ne partageaient pas le point de vue de l'évêque sur cette question $^{33}$.

L'évêque de Saint-Albert exprimait à nouveau sa conviction à l'honorable David Laird, lieutenant-gouverneur des Territoires du NordOuest.

${ }^{29}$ Grandin au Col. James Farquharson McLeod, magistrat stipendiaire du Nord-Ouest, assistant commissioner jusqu'en 1877 de la North West Mounted Police, 3 janvier 1876 (EG 22, 309).

${ }^{30}$ Souvenirs (EG 4, 239) Grandin à $M^{\text {gr }}$ Fabre, ler mai 1879 (EG 8. 278).

${ }^{31}$ Voir, de Gaston Carrière, "Contribution des Oblats de Marie Immaculée de langue française aux études de linguistique et d'ethnologie du Nord Canadien**, Culture 12 (1951) pp. 213-26; "Une riche collection de manuscrits en langues indiennes", Culture 18 (1957) pp. 105-12; “Contribution des missionnaires à la sauvegarde de la culture indienne", Études Oblates 31 (1972) pp. 165-204.

${ }^{32}$ Gaston Carrière, Histoire documentaire de la Congrégation des Missionnaires Oblats de Marie-Immaculée dans l'Est du Canada, 12 vol. Ottawa, 1957-1975, t. 4, p. 48.

${ }^{33}$ Grandin à $M^{\text {gr }}$ Taché, 8 avril 1864 (EG 9, 148). 
Depuis plus de vingt-cinq ans, que je travaille à évangéliser les sauvages du territoire du Nord-Ouest $\mathrm{j}$ 'ai pu acquérir la certitude que le meilleur moyen, j'oserai presque dire l'unique, de faire parmi eux un bien réel et durable, c'est de prendre et de faire siens les petits enfants, on leur fait ainsi oublier les usages et les moeurs de leurs ancêtres. On leur rend la vie nomade impossible, on en fait des hommes civilisés. [ ...] Appuyé sur des faits et sur des expérience de vingtsept ans, je puis assurer que c'est le manque d'éducation qui fait le sauvage. $^{34}$

Le travail de civilisation impliquait la disparition du nomadisme et l'oubli des usages et moeurs indiens et supposait une éducation qui exigeait beaucoup de temps et de patience.

À seize ans, l'enfant en général et l'enfant sauvage surtout, dont l'éducation est plus difficile et plus lente, sera à peine sorti de l'école et n'aura par conséquent pu être formé au travail et à l'économie domestique, ce qui lui importe au moins autant que de savoir lire et écrire; il tombera dans un milieu où l'économie et le travail sont absolument inconnus, ce qui sera d'autant plus pernicieux pour le dit enfant, qu'il a naturellement de la répugnance pour ces vertus domestiques qui ne peuvent lui être inspirées que lentement et difficilement, et il subira d'autant plus les fâcheuses influences sauvages que jusqu'alors il aura été plus retenu. ${ }^{35}$

Devant cette constatation, Grandin souhaitait que les enfants indiens demeurent dans les établissements des missionnaires jusqu'à leur mariage ou la majorité, c'est-à-dire vingt ans.

La grande majorité des enfants du pays, qu'ils gagnent peu ou beaucoup, n'ont jamais rien quand ils se marient et sont dans l'impossibilité de se former le plus petit établissement, je voudrais donc pour le bien de ces enfants, soit qu'ils restent à l'établissement où ils sont engagés ou qu'avec l'agrément du supérieur ils s'engagent ailleurs, qu'ils ne puissent ni recevoir ni administrer le prix de leur travail mais que l'institution l'administrât pour eux jusqu'à leur mariage ou leur majorité. ${ }^{36}$

Grandin voulait transmettre aux jeunes Indiens le sens de l'économie et du travail qui importait autant que de savoir lire et écrire. Pour ce faire, il fallait retirer les enfants de leur milieu «sauvage» et faire en sorte

${ }^{34}$ Grandin à l'hon. Laird, 25 janvier 1880 (EG 22, 192).

${ }^{35}$ Grandin à l'hon. Laird, 2 avril 1880 (EG 22, 196).

${ }^{36}$ Grandin à l'hon. Laird, 2 avril 1880 (EG 22, 196-7). 
qu'ils n'y retournent pas avant leur mariage ou leur majorité. Il n'était pas nécessaire que tous les enfants soient en institution; certains pouvaient être placés dans des familles chrétiennes et civilisées, à certaines conditions.

Je connais pour ma part plusieurs bonnes familles qui adopteraient volontiers un enfant sauvage, mais à quoi bon? se disent-elles, quand nous aurons fait de grands sacrifices pour cet enfant, qu'il pourra nous dédommager un peu, on nous l'enlèvera. Ce qui est vrai pour les familles l'est aussi pour les institutions religieuses. Il faut qu'elles vivent, qu'elles se soutiennent, il faut qu'elles continuent et étendent leurs oeuvres de charité, ne serait-il pas juste que celui qui par leurs soins charitables est capable de travailler et de gagner, les dédommageât de leurs sacrifices et aidât à soutenir les petits qui viennent après lui et ceux qui pour des infirmité physiques et morales ne pourront jamais s'établir et seront toujours à la charge de ces établissements de charité. ${ }^{37}$

L'évêque concluait sa lettre en demandant des lois spéciales pour régler l'adoption des enfants indiens par des institutions ou des familles: donner l'autorité paternelle complète aux institutions jusqu'à la majorité, vingt ans accomplis; permettre à ces institutions et aux familles qui accepteraient d'adopter des enfants de bénéficier d'une partie du travail de ces enfants.

À partir de l'expérience dans l'Est canadien où on n'a pas réussi à faire des Indiens des cultivateurs, $\mathrm{ni}$ à empêcher leur extinction, $\mathbf{M}^{\mathrm{gr}}$ Alexandre Taschereau, archevêque de Québec, fit part de son scepticisme face à l'effort de civilisation des Indiens du Nord-Ouest, $\mathbf{M}^{\mathrm{gr}}$ Grandin répondit :

Je connais dans notre Nord-Ouest un bon nombre de sauvages pur sang qui dès leur enfance ont été recueillis par des familles métisses, et sont aujourd'hui mêlés aux métis, vivant comme eux de leur travail. Un certain nombre de femmes sauvages, élevées dans nos établissements et mariées à des métis, font de dignes épouses et d'excellentes mères de famille. Parmi nos jeunes, ceux que nous avons mis en liberté à l'âge de $\mathbf{1 4}$ ou $\mathbf{1 5}$ ans sont des sans souci qui vont arriver à leur mariage sans un sou devant eux, cependant ils savent travailler, ils se font vivre et peuvent faire vivre leur famille. Je n'ai pas de doute pour ceux que je garde jusqu'à leur mariage. ${ }^{38}$

\footnotetext{
${ }^{37}$ Grandin à l'hon. Laird, 2 avril 1880 (EG 22, 197).

${ }^{38}$ Grandin à Mgr Elzéar-Alexandre Taschereau, archevêque de Québec, 11 décembre 1882 (EG 7, 489).
} 
L'expérience des orphelinats confirmait qu'il était possible d'intégrer les enfants indiens à la population métisse. Grandin croyait en outre toujours possible de faire des Indiens des "chrétiens et des hommes qui puissent vivre de leur travail» dans la société blanche comme le faisaient les Métis: les enfants qu'il avait élevés n'étaient plus "sauvages" mais se mêlaient aux Métis et vivaient comme eux; or, la civilisation des Métis, si elle n'arrivait pas à la hauteur des habitants des villes, valait bien celle de beaucoup de paysans de France ${ }^{39}$. Pour lui, il y avait donc civilisation même dans le cas de la culture intermédiaire des Métis.

Pour l'évêque de Saint-Albert, "civilisation" signifiait également francisation ou anglicisation. Il visait à transformer les enfants indiens en petits Français ${ }^{40}$.

Puisque nous faisons tant que d'élever des enfants sauvages et que nous en faisons, je puis dire, des hommes aussi civilisés que les Métis et que beaucoup d'étrangers les méprisent nous ne voudrions pas que leur nom rappelât plus tard leur origine et ne fût une humiliation pour leurs descendants, pour cette raison nous leur donnons des noms de famille anglaise ou française.

Ajoutez que les noms sauvages sont parfois des plus ridicules et même dégoûtants. $[\ldots]$ En leur faisant porter de semblables noms, les employés du Gouvernement comprendraient mieux sans doute que ce sont des enfants sauvages, mais ces pauvres enfants seraient tournés en ridicule par leurs compagnons et leurs descendants seraient humiliés. ${ }^{41}$

$\mathbf{M}^{\mathrm{gr}}$ Grandin cherchait donc à franciser les petits Indiens même dans leur nom pour leur faire oublier leur origine et pour la faire oublier à ceux qu'ils côtoieraient.

Voilà ce qu'il entend par civilisation: former aux valeurs d'économie domestique et de travail surtout agricole, être en mesure de vivre de son travail en connaissant un métier ou l'autre.

\footnotetext{
${ }^{39}$ Grandin à l'Archevêque de Québec et aux Évêques de la Province de Québec, 29 janviers 1883 (EG 7, 496-502).

${ }^{40}$ Grandin à l'Évêque de Laval, Jules Denis Le Hardy du Marais, 18 avril 1880 (EG 8, 326); Grandin à $\mathbf{M}^{\mathrm{gr}}$ Jules François LeCoq, évêque de Nantes, 28 janvier 1881 (EG 8, 367).

${ }^{41}$ Grandin à M. Alexander Galt, assistant commissioner of Indian Affairs, ancien ministre des Finances et "high commissioner" à Londres, s.d. (EG 22, 133).
} 
Dès le début de son ministère, Grandin adopta de petits garçons indiens. Les soeurs Grises de Montréal ouvrirent dès leur arrivée écoles et orphelinats: Ile-à-la-Crosse en 1860, Lac-La-Biche en 1862 et SaintAlbert en $1863^{42}$. Les Indiens s'y révélaient intelligents et capables d'apprendre mais il fallait tenir compte de leurs besoins particuliers.

Nous avons remarqué que les enfants montagnais sont très susceptibles d'instruction; mais il ne faut pas les retirer tout à fait de la vie sauvage. Ils meurent même dans nos établissements, si nous ne leur accordons le grand air, certains travaux extérieurs et la nourriture du pays. ${ }^{43}$

En 1872, Grandin fit part du succès des religieuses avec leurs écoles.

Des enfants sauvages élevés par les soeurs et par nous, $[\ldots]$ n'ont plus rien de sauvage que le sang; ils sont instruits convenablement de leur religion surtout et plusieurs peuvent parler et écrire le français assez correctement. ${ }^{44}$

Jusqu'à cette époque, seuls les orphelins et quelques enfants pendant l'hiver allaient à l'école; à partir de ce moment, les Métis commencèrent à comprendre l'importance de l'instruction ${ }^{45}$.

Un des facteurs importants de civilisation des Indiens étaient la présence d'enfants métis dans les orphelinats et écoles catholiques.

Les enfants métis s'y trouvaient mêlés aux enfants sauvages, ces derniers bénéficiaient plus de ce contact que les autres n'y pouvaient perdre. [...] Presque tous nos enfants sauvages élevés dans nos établissements se mêlaient aux Métis et on ne les distinguait pas de ces derniers; ce n'était pas la civilisation complète mais au moins c'était un bon commencement. ${ }^{46}$

Quelques rapports nous permettent d'avoir une idée du nombre d'enfants éduqués dans les orphelinats et les écoles de mission. À la fin de 1876, on comptait cinquante-huit enfants reçus par la mission et quarante-cinq enfants indiens fréquentaient l'école. En 1880, on dénombrait

\footnotetext{
${ }^{42}$ Lettre circulaire, 5 mars 1900 (EG 17, 522-3). Voir Pierre Duchaussois, Femmes Héroiques. Les Soeurs Grises Canadiennes aux Glaces Polaires, Paris, 1927, pp. 42-53.

${ }^{43}$ Note sur $M^{\mathrm{gr}}$ Taché pour Dom Paul Benoît, son biographe (EG 5, 299).

${ }^{44}$ Grandin au P. Aubert, 7 décembre 1872 (EG 14, 548 ).

${ }^{45}$ Grandin au P. Aubert, 7 décembre 1872 (EG 14, 538): Notes de $M^{g r}$ Grandin sur l'Église du Nord-Ouest (EG 6, 161 ).

${ }^{46}$ Souvenirs (EG 4, 252).
} 
deux cents jeunes Indiens dans les écoles ${ }^{47}$. Mais les résultats n'étaient pas toujours durables lorsque les enfants retournaient chez leurs parents.

Ces jeunes gens à l'âge de 13 et 20 ans sortent de nos établissements pour retourner dans leur famille. Ils se trouvent isolés au milieu de barbares auxquels ils ne peuvent être indifférents ce sont leurs pères, leurs parents, à tous les degrés qui se moquent de leurs usages, et leur font vite reprendre les usages nationaux. Cet enfant se laisse d'autant plus vite prendre qu'il n'a qu'à céder à ses penchants naturcls, il est libre; on lui dit qu'il ne peut fairc autrement que ses parents et amis. ${ }^{48}$

Pour Grandin, le retour sur les réserves ne pouvait contribuer à maintenir le degré de "civilisation" atteint dans les écoles. Ce dernier suggérait la création de réserves spéciales pour ses anciens élèves.

Pour que ces enfants puissent conserver les fruits de leur éducation, il faudrait qu'ils fussent assez nombreux pour faire bande à part. [...] J'avais demandé au Gouvernement une réserve spéciale pour nos enfants où nous les placerions après leur mariage, sur lesquels nous aurions certainement une certaine influence, je suis certain qu'à ces conditions nous réussirions mieux. ${ }^{49}$

Lors d'un voyage en France, l'évêque de Saint-Albert visita un pénitencier pour jeunes détenus, à Citeaux, dont l'organisation correspondait à ce dont il rêvait pour civiliser les petits Indiens.

J'achève après le déjeuner de visiter l'établissement, les ateliers, je n'ai encore rien vu d'aussi achevé dans le genre, je vois à la lettre réalisé ce que je rêve de faire avec les sauvages. [...] J'ai vu ces petits au travail, suivant leurs forces, tous absolument travaillent des mains et étudient tous les jours; les progrès en tout en sont bien plus marqués. ${ }^{50}$

L'idée des «écoles industrielles» se voyait confirmée chez Grandin. C'est avec ce projet qu'il retournait au Canada en 1879.

\footnotetext{
${ }^{47}$ Grandin à M. l'abbé de Girardin, directeur de la Sainte-Enfance, fin 1876 (EG 16, 533); Rapport au Card. Simeoni, 20 octobre 1880 (EG 7, 414).

${ }^{48}$ Souvenirs (EG 4, 252).

${ }^{49}$ Souvenirs (EG 4, 252-3). Un plan de ce genre fut réalisé à l'école de Lebret. Voir Rudolph Nowakowski, Indian Residential Schools in Saskatchewan Conducted by the oblate Fathers, Thèse de maîtrise à l'Institut de Missiologie de l'Université d'Ottawa, Ottawa, 1962, pp. 90-1.

${ }^{50}$ Journal, 12 avril 1878 (EG 1, 132); sur les écoles industrielles, voir Nowakowski, Indian Residential Schools.
} 
Jugez vous-mêmes du bien que feraient $[\ldots]$ ces écoles industrielles et fermes modèles où un grand nombre d'enfants sauvages seraient formés à la vie chrétienne et civilisée, au travail, à l'économie domestique, etc. Leur exemple, comme à Saint-Albert, serait d'une bienfaisante influence auprès des autres enfants et des adultes eux-mêmes; une fois mariés, ils seraient autant de maîtres-colons dans leur nation et avant 100 ans les sauvages auraient disparu comme sauvages mais ils vivraient comme peuples civilisés, ils seraient utiles au pays et pourraient faire partie de la société. ${ }^{51}$

Mais, sans ce projet, Grandin croyait que l'avenir était sombre pour les Indiens.

Réduits à nos seules forces, grâce à notre dévouement et abnégation sans bornes, nous procurerons, comme nous l'avons fait jusqu'à présent les avantages de la civilisation à quelques sujets seulement, mais cela ne sauvera pas les sauvages en général, dans 100 ans peut-être, il n'en existera plus guère, ils ne seront pas transformés en hommes civilisés; le jeûne, les misères physiques et morales les auront tuées et fait disparaître mais en attendant, ils ne seront pas sans occasionner bien des désagréments aux Blancs du pays et seront pour le Gouvernement une bien [... ] lourde charge. 52

Nowakowski attribue à l'évêque de Saint-Albert l'origine de l'idée d'écoles industrielles pour les Indiens. Dans les années 1880, Grandin, Taché et Lacombe approchèrent le Gouvernement canadien pour obtenir des écoles de ce type sous le contrôle temporel de l'État mais sous la direction spirituelle des Oblats et des religieuses ${ }^{53}$. À l'été 1883 , le Gouvernement autorisait l'établissement de trois écoles industrielles, une protestante, à Battleford, et deux catholiques, à Qu'Appelle et à Calgary; cette dernière, en faveur dess Pieds-Noirs, était située dans le diocèse de Saint-Albert et le P. Lacombe en devenait le premier principal ${ }^{54}$. Le personnel de l'école industrielle devait être comme suit:

un principal, un fermier, un charpentier, un maître d'école, un cuisinier et une matrone, tous retribués par le Gouvernement [...] Plus tard un forgeron, un cordonnier, ou autres artisans pourront faire partie du personnel enseignant. ${ }^{55}$

\footnotetext{
${ }^{51}$ Grandin au P. Joseph Lestanc, consulteur, 3 avril 1880 (EG 16, 382).

52 Grandin au P. Lestanc, 3 avvril 1880 (EG 16, 382).

${ }^{53}$ Nowakowski, Indian Residential Schools, pp. 29-30; 34-5.

54 Voir Actes des Délibérations du Conseil du Vicariat de la Saskatchewan, 10 septembre 1883 (EG 15, 144); Nowakowski, Indian Residential Schools, pp. 35-6.

${ }^{55}$ Actes des Délibérations, 10 septembre 1883 (EG 15, 144).
} 
Quant aux enfants qu'on devait y accueillir, il avait été décidé ce qui suit:

Le nombre des enfants ne doit pas actuellement dépasser le nombre de trente. $[\ldots]$ Il est reconnu par tous que pour réussir, il faut prendre ces enfants aussi jeunes que possible; 5 ou 6 ans serait la meilleure âge. Autant que possible, on s'en tiendra là, sans pourtant exclure les enfants de 7, 8 ou 9 ans qui donneraient les garanties suffisantes. ${ }^{56}$

On insistait, de plus, pour ne prendre que de jeunes enfants encore peu marqués par la culture indienne. Le P. Lacombe, principal de l'école, rencontra des difficultés dans cette nouvelle oeuvre mais son supérieur l'encourageait.

Je crois comprendre que malgré toutes vos difficultés, il ne faut pas absolument désespérer de votre oeuvre, puisque vous reconnaissez que vos jeunes Pieds-Noirs se livrent volontiers à l'étude, aiment à imiter les blancs et à prendre leurs manières. ${ }^{57}$

Les jeunes Pieds-Noirs semblaient aimer l'étude et la manière de faire des Blancs, Pourtant le succès était loin d'être celui espéré. Grandin analysait les difficultés.

J'ai toujours cru que l'on voulait aller trop vite avec cette école, si au lieu de 20 ou 30 enfants dans le principe vous n'en aviez pris que 8 ou 10 tout petits et une fois formés à la discipline, vous eussiez pu en prendre d'autres qui se fussent formés sans difficultés à l'exemple des autres. Si le Gouvernement tient absolument à en avoir 30 de suite, pourquoi ne pas achever ce nombre avec des enfants d'une nation différente? Vous avez autour de vous des Métis plus dégradés que bien des sauvages, si on ne veut pas des Métis, qu'on prenne des sauvages étrangers aux Pieds-Noirs et plus faciles à soumettre, ce qui rend vos sauvageons si exigeants et si difficiles à dompter, c'est d'abord je crois parce que vous en avez pris de trop grands et que tous ensemble ils font des partis qu'ils ne feraient pas s'ils étaient plus mêlés à des étrangers. ${ }^{58}$

Les enfants pieds-noirs étaient difficiles à soumettre, à dompter et plus indépendants que les autres. Grandin croyait préférable de les prendre très jeunes et d'avoir dans la même école des enfants des différentes nations indiennes et même des Métis si c'était possible, comme c'était le cas à Saint-Albert et à l'Ile-à-la-Crosse.

\footnotetext{
${ }^{56}$ Actes des Délibérations, 10 septembre 1883 (EG 15,144 et 146).

${ }^{57}$ Grandin au P. Albert Lacombe, missionnaire, 9 janvier 1885 (EG 15, 401 ).

${ }^{58}$ Grandin au P. Lacombe, 12 mars 1885 (EG 15, 407).
} 
Sa perception était encore assez sombre au printemps 1889 .

Leur [les missionnaires] grande occupation est de soigner les petits enfants, mais quelle corvée avec des parents qui ne s'y prêtent nullement. S'ils peuvent former une génération moins grossière, j'oserais dire moins animale que la génération actuelle, avec le temps nos successeurs finiront par les christianiser peut-être. S'ils ne meurent pas avant car l'immoralité qui a beaucoup augmenté depuis que les blancs se sont multipliés pourraient bien les faire disparaître comme nation, si au moins nous pouvions les convertir avant. ${ }^{59}$

Après une visite des écoles, quelques mois plus tard, il paraît plus optimiste.

J'ai visité leurs écoles et je ne puis moins faire que d'en espérer d'heureux résultats. Malheureusement ces enfants en sortant de l'école subissent toujours l'influence de la famille et de la nation, mais il restera quelque chose de l'influence du prêtre surtout s'il peut les suivre et peu à peu les moeurs s'adouciront. ${ }^{60}$

Le succès demeurait pourtant très limité avec les enfants devenus grands. En 1892, pas un seul n'avait pu être gardé une fois devenu un peu grand sauf Baptiste Pépin ${ }^{61}$.

Une des difficultés des écoles industrielles soutenues par le Gouvernement fut l'absence d'enfants métis, puisque ceux-ci n'avaient pas fait de traités avec le Canada; or, pour Grandin, ce contact indien-métis était un grand facteur de civilisation ${ }^{62}$. Cette idée de civilisation des Indiens par les petits enfants survécut à l'évêque de Saint-Albert puisque les Oblats furent engagés dans les écoles industrielles puis résidentielles jusqu'à récemment.

Pour Grandin, qui constatait l'essai infructueux auprès des adultes, l'efffort civilisateur des missionnaires devait se diriger vers les petits enfants métis et surtout indiens. En les prenant tout petits, il était possible de faire disparaître en eux le genre de vie «sauvage», leurs usages et les moeurs, même leur langue maternelle pour les amener à une cul-

\footnotetext{
${ }^{59}$ Grandin au P. Fabre, 6 avril 1889 (EG 13, 502-3).

${ }^{60}$ Grandin au P. Fabre, 22 novembre 1889 (EG 13, 533).

${ }^{61}$ Voir Journal, 31 décembre 1892 (EG 2, 404). Baptiste Pépin accompagna Grandin dans ses voyages au Mackenzie. Voir Adrien G. Morice, Dictionnaire historique des Canadiens et des Métis français de l'Ouest, Québec-Montréal-Winnipeg, 1912, pp. 225-6.

${ }^{62}$ Souvenirs (EG 4, 252).
} 
ture intermédiaire semblable à celle des Métis. Pour ce faire, il était nécessaire de prendre les enfants très jeunes et de les garder dans les établissements de la mission jusqu'à leur majorité ou leur mariage.

On visait à transmettre aux jeunes Indiens les valeurs de l'économie et du travail qui leur permettraient de vivre dans la société occidentale. Le retour chez les parents montrait comment peu profond pouvait être l'éducation transmise. L'évêque de Saint-Albert suggérait donc la création de réserves spéciales où les jeunes Indiens seraient encouragés à garder ce qu'ils avaient acquis et à vivre avec les Métis de qui ils s'étaient rapprochés par l'éducation. Il proposait également la création des écoles industrielles où les enfants seraient initiés aux différents métiers : travail agricole, menuiserie, etc.

Si Grandin fit état de succès auprès d'un certain nombre d'Indiens reçus à la mission, les enfants pieds-noirs furent particulièrement difficiles à mener et peu d'enfants des différentes nations demeurèrent dans les établissements de la mission une fois devenus grands.

Claude Champagne

Institut des Sciences Missionnaires

Université Saint-Paul (Ottawa) 


\section{Rockies}

Comme, redescendus des hauteurs de l'air où les hommes commandent leurs nacelles,

Toutes plaines dévorées sans les voir, - nous roulions sur les traces du soleil,

Le Grand Déversement de rocs poussés hors du flanc de la terre par Yahweh

Commença d'apparaître sous la steppe : ondulation pareille à la mer Quand l'attrait de la lune prépare son déferlement sur les rivages.

Là-bas, drapée en bure et surcot de neige, allongée, immobile, sans face, La Montagne, sur l'azur mordoré, découpe son mouvement de pierre, La ligne en creux de son usure telle qu'on imagine un temple Aux gradins énormes surmontés d'un autel qui touche l'assise du ciel. Aujourd'hui, tant d'âges cumulés sur l'abîme en poussière, en limaille, en atomes,

Sous la force de l'eau torrentielle, du vent largue et des laminoirs de glace,

Ont réduit la montagne au dessin des neumes sur la page du missel :

Il monte, décline un peu, remonte et, par bonds, de palier en palier dépasse

Les lignes de portée. Elle chante un hymne visible, que le Créateur écoute

Et nous nous taisons pour l 'entendre. 